\title{
Medical and surgical outcome of tricuspid regurgitation caused by flail leaflets
}

\author{
David Messika-Zeitoun, MD \\ Helen Thomson, MD \\ Michael Bellamy, MDa \\ Christopher Scott, MS ${ }^{b}$ \\ Christophe Tribouilloy, MD \\ Joseph Dearani, $\mathrm{MD}^{\mathrm{C}}$ \\ A. Jamil Tajik, MD \\ Hartzell Schaff, MD \\ Maurice Enriquez-Sarano, MD
}

Objective: We sought to evaluate the medical and surgical outcome of tricuspid regurgitation caused by flail leaflets.

Methods: We analyzed the cause, clinical presentation, outcome, and natural history of 60 patients with tricuspid regurgitation caused by flail leaflets, a cause of mostly severe and organic tricuspid regurgitation, diagnosed by means of echocardiography between 1980 and 2000.

Results: The main cause was traumatic $(62 \%)$. Clinical presentation was often severe: $57 \%$ were symptomatic, $33 \%$ had a history of congestive heart failure, and $40 \%$ had a history of atrial fibrillation. Compared with expected survival of the US matched population, excess mortality $(39 \% \pm 10 \%$ at 10 years or $4.5 \%$ yearly, $P<$ .01) was observed. Even patients asymptomatic at presentation experienced high tricuspid-related event rates (at 10 years, $75 \% \pm 15 \%$ had symptoms or heart failure, atrial fibrillation, surgical intervention, or death). In those patients severe enlargement of right-sided chambers was predictive of poor outcome (at 5 years: $86 \% \pm 9 \%$ vs $39 \% \pm 11 \%, P<.01)$ independent of cause $(P=.31)$. The poor medical outcome was further confirmed by high event rates $(69 \% \pm 9 \%$ at 15 years) in the natural history beginning from the flail's occurrence. Tricuspid operations were performed in 33 patients $(55 \% \pm 7 \%$ at 5 years), with valve repair in $82 \%$, low mortality $(3 \%)$, and, despite frequently refractory atrial fibrillation, symptomatic improvement in $88 \%$.

Conclusion: Tricuspid regurgitation caused by flail leaflets is a serious disease associated with excess mortality and high morbidity. Tricuspid valve repair can often be performed with low risk, allowing symptomatic improvement. These results suggest that surgical intervention should be considered early in the course of the disease before the occurrence of irreversible consequences.

Address for reprints: Maurice EnriquezSarano, MD, Division of Cardiovascular Diseases and Internal Medicine, Mayo Clinic, 200 First St SW, Rochester, MN 55905 (E-mail: sarano.maurice@mayo. edu).

J Thorac Cardiovasc Surg 2004;128: 296-302

$0022-5223 / \$ 30.00$

Copyright () 2003 by The American Association for Thoracic Surgery

doi:10.1016/j.jtcvs.2004.01.035

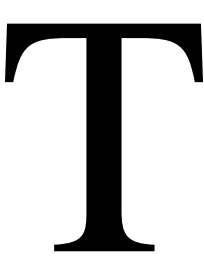

ricuspid regurgitation (TR) is a common Doppler echocardiographic finding ${ }^{1}$ resulting either from intrinsic valve abnormalities (organic TR) or functional malcoaptation of structurally normal valves. Despite this high prevalence, the effect of TR on clinical outcome remains elusive for several reasons. First, mild TR is frequent even in normal subjects, but severe TR is less frequent. Second, TR is difficult to assess, and quantitative methods are only recently available. ${ }^{2}$ Third, the prognostic significance of severe TR has been mainly evaluated in functional regurgitation. ${ }^{3,4}$ Functional TR is most common but is dynamic and changes with 
the pulmonary pressure, and its outcome reflects the cause and severity of the underlying pulmonary hypertension responsible for the regurgitation. In contrast, organic TR is due to permanent and organic valvular lesions and might allow specific assessment of the outcome of the TR with minimal confounding factors. Complicated courses with intractable heart failure have been described, ${ }^{5-7}$ but other reports stressed the fact that patients with severe TR can do well for prolonged periods of time. ${ }^{8,9}$ Furthermore, the tricuspid valve can be completely removed with reportedly good long-term clinical tolerance, ${ }^{10,11}$ so that TR, even of a marked degree, has the reputation of being well tolerated. Because of these contradictory findings and the paucity of natural history studies, the effect of severe TR on outcome remains uncertain. This uncertainty is an impediment to the clinical management of patients with TR, as emphasized in most current practice guidelines, and although this condition can be treated with surgical intervention, operative indications are not clearly defined. ${ }^{12}$

TR caused by flail leaflets is a permanent, organic, and mostly severe $\mathrm{TR}^{13}$ and is reliably diagnosed by means of 2-dimensional echocardiography. ${ }^{14}$ However, there has been no comprehensive study, and the experience regarding this valve disease is limited to case reports or short series involving less than 25 patients. ${ }^{13,15,16}$ Accordingly, we examined the causes, clinical presentations, and outcomes of patients with tricuspid flail leaflets.

\section{Methods \\ Study Population}

Patients were included if they had tricuspid flail leaflets diagnosed by means of 2-dimensional echocardiography between 1980 and 2000 in our institution. The cause of the valve disease was defined on the basis of history and echocardiographic and surgical findings if available. A history of severe chest trauma was required for the diagnosis of traumatic (noniatrogenic) flail. Symptoms were classified according to the New York Heart Association grading. Congestive heart failure was defined by using the Framingham criteria, ${ }^{17}$ and recurrent episodes were noted. The TR was clinically assessed on the basis of the presence of systolic murmur and hepatic or jugular venous pulsatility. Patients were divided into 3 groups according to functional status. Symptomatic patients (NHYA class $\geq$ II, congestive heart failure, or both) were included in group I if there was no other reason for symptoms than TR and in group II if associated conditions could have played a role in symptoms. Asymptomatic patients (NHYA class I and no history of congestive heart failure) formed group III. The diagnosis of atrial fibrillation (AF) required electrocardiographic confirmation. $\mathrm{AF}$ that either preceded the known occurrence of TR or was due to another cause was not considered as related to TR. Follow-up information was obtained through clinical records, postal surveys, death certificates, and telephone calls to patients, relatives, or physicians. If possible, the date of occurrence of TR was determined on the basis of clinical information, medical records, and echocardiographic examinations.

\section{Echocardiographic Methods}

Comprehensive transthoracic echocardiography was consistently performed throughout the study period. The diagnosis of flail leaflet was based on failure of leaflet coaptation with rapid systolic movement of the involved leaflet tip in the right atrium. ${ }^{5,18}$ TR was assessed semiquantitatively in 4 grades (mild, moderate, moderate to severe, and severe) on the basis of comprehensive assessment by using pulsed-wave Doppler, color jet extent, hepatic venous flow reversal, or quantitative methods. ${ }^{2,19}$ Assessment of right atrial and ventricular dilatation was performed on the basis of a comprehensive examination of these cavities from multiple views and classified into 4 grades (absent, mild, moderate, and severe) by experienced physicians. The size of other cardiac chambers provided useful references for this assessment. Severe enlargement of rightsided chambers was defined by a severe dilatation of the right atrium, the right ventricle, or both cavities. Additionally, endsystolic right atrial and end-diastolic right ventricular areas were planimetered from a single-plane, apical, 4-chamber view to compare right atrial and ventricular sizes between groups. Right ventricular fractional area contraction was calculated. Areas were normalized to body surface area and compared with those of 23 other patients with functional (nonflail) TR of mild or at most moderate degree, as reference values.

\section{Statistical Analysis}

Data are presented as means $\pm \mathrm{SD}$ or percentages. Comparisons between groups used analysis of variance, $t$ tests, or $\chi^{2}$ tests as appropriate. The end points analyzed were total mortality, occurrence of symptoms or heart failure, occurrence of new AF (paroxysmal or chronic), and the composite end point of symptoms, new AF, tricuspid surgery, or death. The clinical outcome after diagnosis was analyzed overall and in subsets stratified by symptomatic status at diagnosis. Patients operated on within 3 months of diagnosis were considered as initially surgically managed, whereas the other patients were considered initially conservatively managed. The rates of mortality and of other end points were estimated by using the Kaplan-Meier method, and differences between groups were tested with the 2-sample log-rank test. Survival was compared with the expected survival of the age- and sex-matched white 1990 US population and tested with the 1-sample log-rank test. Cox proportional-hazards analysis was performed to evaluate predictors of outcome. The end points were analyzed starting at diagnosis for analysis of clinical outcome after diagnosis or starting at the date of occurrence of TR for analysis of natural history of the disease.

\section{Results}

\section{Cause and Clinical Presentation of Tricuspid Flail Leaflets}

Between 1980 and 2000, 60 patients with tricuspid flail leaflets were identified by means of echocardiography. The cause was mainly traumatic $(n=37$ [62\%]), related to blunt chest trauma (mostly motor vehicle accident) in 19 and to iatrogenic chordal severing (mostly right ventricular biopsy) in 18. Nontraumatic causes $(\mathrm{n}=23$ [38\%]) were myxomatous $(\mathrm{n}=7[12 \%])$, infective endocarditis $(\mathrm{n}=5[8 \%])$, and congenital $(\mathrm{n}=2[3 \%])$, and in $9(15 \%)$ patients with 
TABLE 1. Baseline clinical and echocardiographic characteristics of patients with tricuspid regurgitation caused by flail leaflets

\begin{tabular}{|c|c|c|c|c|c|}
\hline \multirow[b]{2}{*}{ Variables } & \multirow[b]{2}{*}{$\begin{array}{c}\text { Overall } \\
\text { population }\end{array}$} & \multicolumn{3}{|c|}{ Baseline characteristics by group } & \multirow[b]{2}{*}{$P$ value } \\
\hline & & $\begin{array}{c}\text { Group I } \\
\text { (symptomatic } \\
\text { because of TR) }\end{array}$ & $\begin{array}{l}\text { Group II (symptomatic } \\
\text { with associated } \\
\text { diseases) }\end{array}$ & $\begin{array}{c}\text { Group III } \\
\text { (asymptomatic) }\end{array}$ & \\
\hline $\mathrm{N}$ & 60 & 23 & 11 & 26 & \\
\hline Age (y) & $51 \pm 26$ & $50 \pm 21$ & $46 \pm 37$ & $55 \pm 25$ & .58 \\
\hline Male sex $(\%)$ & 62 & 48 & 64 & 73 & .19 \\
\hline \multicolumn{6}{|l|}{ Cause $(\%)$} \\
\hline Traumatic, noniatrogenic & 32 & 61 & 18 & 12 & $<.01$ \\
\hline Traumatic, iatrogenic & 30 & 4 & 36 & 50 & \\
\hline History of atrial fibrillation (\%) & 40 & 52 & 64 & 19 & .01 \\
\hline Atrial fibrillation at presentation (\%) & 25 & 30 & 45 & 12 & .03 \\
\hline History of congestive heart failure (\%) & 33 & 48 & 82 & 0 & $<.01$ \\
\hline Recurrent congestive heart failure (\%) & 20 & 35 & 36 & 0 & $<.01$ \\
\hline Severe TR $(\%)$ & 82 & 96 & 82 & 69 & .06 \\
\hline Right atrial area index $\left(\mathrm{cm}^{2} / \mathrm{m}^{2}\right)$ & $21 \pm 10$ & $27 \pm 11$ & $25 \pm 9$ & $16 \pm 6$ & .01 \\
\hline Right ventricular area index $\left(\mathrm{cm}^{2} / \mathrm{m}^{2}\right)$ & $23 \pm 10$ & $25 \pm 8$ & $28 \pm 15$ & $19 \pm 8$ & .04 \\
\hline Severe right-sided chamber enlargement & 58 & 83 & 82 & 27 & $<.01$ \\
\hline Right ventricular fractional area contraction (\%) & $41 \pm 12$ & $38 \pm 17$ & $43 \pm 8$ & $43 \pm 8$ & .37 \\
\hline
\end{tabular}

Values are percentages of patients or means \pm SD.

$T R$, Tricuspid regurgitation.

ruptured tricuspid chordae, no specific cause could be defined, and these patients were considered idiopathic.

At diagnosis (Table 1), age was $51 \pm 26$ years, 37 patients were men, 34 (57\%) were symptomatic (New York Heart Association class $\geq \mathrm{II}), 20$ (33\%) had a history of congestive heart failure, and $12(20 \%)$ had recurrent congestive heart failure. In 2 patients a right-to-left shunt through a patent foramen ovale contributed to hypoxemia. Twenty-four (40\%) patients had a history of AF, and 15 $(25 \%)$ were in AF at presentation. The 6 patients with a history of preexisting chronic or paroxysmal AF unrelated to the TR were excluded from subsequent analysis regarding rhythm disturbance end points.

The flail segment involved the tricuspid anterior leaflet in $33(55 \%)$ patients, the septal leaflet in $16(27 \%)$ patients, the posterior leaflet in $4(7 \%)$ patients, and both the septal and posterior leaflets in $1(1 \%)$ patient, and it could not be clearly defined in $6(10 \%)$ patients. TR was assessed in all patients clinically, in 56 by means of Doppler echocardiography and in 13 by means of catheterization, and was considered moderate in $5(8 \%)$ patients, moderate to severe in $6(10 \%)$ patients, and severe in $49(82 \%)$ patients. TR velocity was $2.4 \pm 0.7 \mathrm{~m} / \mathrm{s}$, which is consistent with normal pulmonary pressures. Thirty-five (58\%) patients had severe enlargement of right-sided chambers. Right atrial and ventricular areas, measurable in 56 patients, were significantly enlarged compared with those in the 23 patients (age, $64 \pm$ 15 years) with mild-to-moderate functional TR $(21 \pm 10 \mathrm{vs}$ $14 \pm 5 \mathrm{~cm}^{2} / \mathrm{m}^{2}, P<.001$, and $23 \pm 10$ vs $16 \pm 6 \mathrm{~cm}^{2} / \mathrm{m}^{2}$, $P<.005$, respectively).
Group I (symptoms caused by TR) was composed of 23 patients, group II (symptoms with associated disease) was composed of 11 patients, and group III (asymptomatic) was composed of 26 patients. Two patients in group I had a normally functioning mitral valve prosthesis. Associated diseases in group II were restrictive cardiomyopathy $(\mathrm{n}=$ $3)$, congenital heart disease $(n=3)$, chronic obstructive pulmonary disease $(n=2)$, left ventricular to right atrial fistula $(\mathrm{n}=1)$, left ventricular dysfunction $(\mathrm{n}=1)$, and lymphoma $(\mathrm{n}=1)$. Nine patients had previous heart transplantation without significant signs of rejection and were asymptomatic (group III). There were no age or sex differences between groups (Table 1), but patients in Group III had less frequent AF or severe TR. Patients in group III had smaller right-sided chamber sizes. In group III only $27 \%$ had severe right-sided chamber enlargement, and right atrial and ventricular areas were smaller than those in group I and II patients but not different from those of control subjects $\left(16 \pm 6\right.$ vs $14 \pm 5 \mathrm{~cm}^{2} / \mathrm{m}^{2}, P=.17$, and $19 \pm 8$ vs $16 \pm$ $\left.6 \mathrm{~cm}^{2} / \mathrm{m}^{2}, P=.12\right)$.

\section{Clinical Outcome}

Follow-up was complete up to 2001 or death in $95 \%$. Mean follow-up time after diagnosis was $5.8 \pm 4.8$ years and was not different between groups $(5.8 \pm 5.4,3.9 \pm 3.5$, and 6.5 \pm 4.8 years, respectively; $P=.37$ ).

Mortality. Fifteen deaths occurred during follow-up, corresponding to a mortality of $39 \% \pm 10 \%$ at 10 years or $4.5 \%$ yearly, which was higher than expected in the US population $(P<.01$; Figure $1, A)$. Seven deaths were 

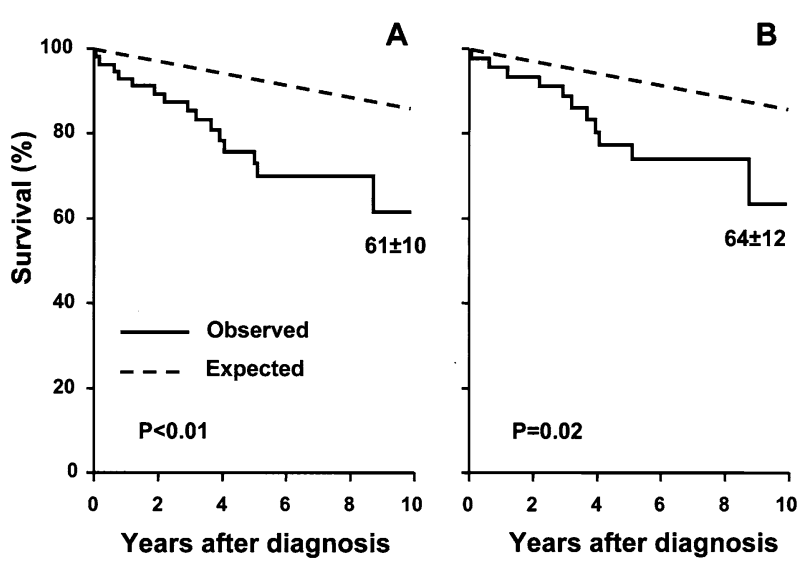

Figure 1. Long-term survival after diagnosis of TR caused by flail leaflets compared with expected survival in the US matched population. Excess mortality $(4.5 \%$ yearly, $P<.01)$ was observed in the overall population ( $n=60$ ) with tricuspid flail leaflets (A) and after exclusion of patients with associated diseases contributing to symptoms (B; $3.8 \%$ yearly, $P=.02$ ).

definitely or partially related to TR (CHF or cardiogenic shock in 6 patients and infective endocarditis in 1 patient), and only 1 posttransplantation patient died in refractory heart failure caused by TR before scheduled surgical repair (without any sign of significant rejection). Excluding patients from group II in whom associated diseases could have affected survival, excess mortality in comparison with that in the general population was also observed $(36 \% \pm 12 \%$ at 10 years or $3.8 \%$ yearly, $P=.02$; Figure $1, B)$, and 5 of 11 deaths were related to TR.

Surgery. Tricuspid surgery was performed in 33 patients (55\% $\pm 7 \%$ at 5 years), confirming the flail leaflet in all cases. Indications were symptoms in $28(85 \%)$ patients, physician's preference in $4(12 \%)$ patients, and associated coronary disease requiring surgical intervention in $1(3 \%)$ patient. There was no emergency procedure, but 23 interventions were performed within 3 months of diagnosis, and 30 were performed at our institution. The tricuspid valve was repaired in $27(82 \%)$ patients and replaced in $6(18 \%)$ patients. Surgical repair consisted mainly of placation-resection of the flail segment. Annular dilatation was corrected with a De Vega annuloplasty $(n=16)$ or a Carpentier tricuspid ring $(\mathrm{n}=7)$. Subvalvular support by artificial chordae was used in 4 patients. Tricuspid prostheses consisted of 4 Carpentier-Edwards bioprotheses, 1 Saint-Jude, and 1 Starr-Edwards. Additional procedures performed were reduction right atrioplasty (52\%), closure of a shunt at the atrial level $(45 \%)$, and coronary bypass grafting $(6 \%)$. One patient had an aortic valve replacement for moderate aortic stenosis, and 1 patient had an obliteration of a posttraumatic Valsalva aneurysm, with resuspension of the aortic valve. Operative mortality occurred in 1 (3\%) patient

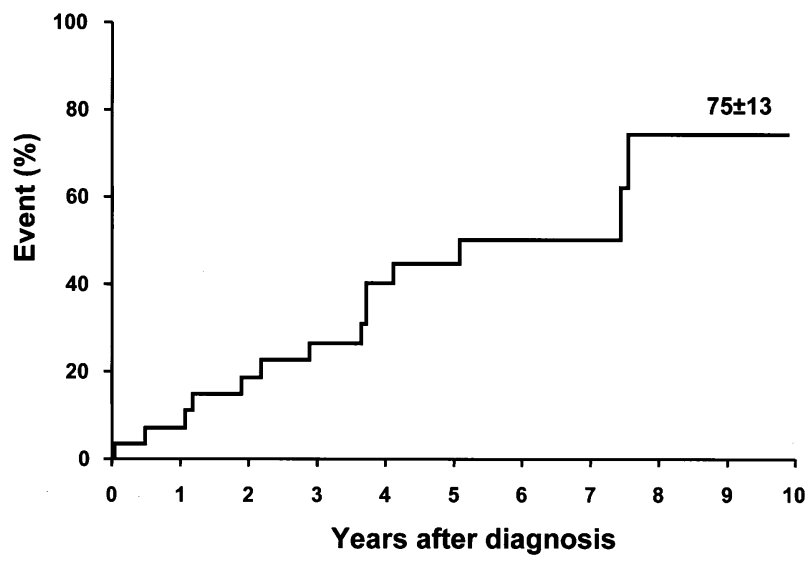

Figure 2. Clinical outcome after diagnosis of TR caused by flail leaflets of asymptomatic patients. The Kaplan-Meier curve depicts the incidence of the combined end point of symptoms or heart failure, new AF, cardiac surgery, or death.

with associated coronary bypass because of sepsis and multiorgan failure. Only 2 (7\%) of 27 patients undergoing repair had residual TR of more than mild degree at predischarge assessment. Symptomatic improvement was observed in $88 \%$, but patients in AF before the operation remained so, and among those in sinus rhythm, the incidence of new AF (paroxysmal or chronic) was $32 \% \pm 10 \%$ at 5 years and $45 \% \pm 12 \%$ at 10 years ( $8.4 \%$ yearly).

Analyzed by group, 20 of 23 patients in group I underwent tricuspid surgery. The 3 others were conservatively managed, 2 because of advanced age, and 1 died before scheduled surgical intervention. Five patients of group II also underwent surgical repair, and 6 were conservatively managed because of associated diseases. Eight patients of group III (asymptomatic) underwent tricuspid surgery indicated because of physician's preference in 4 , new symptoms in 3 , and associated coronary disease in 1 .

Clinical outcome in asymptomatic patients. Fifteen patients of group III (58\%) experienced symptoms, heart failure, new AF, surgical intervention, or died during followup. Ten years after diagnosis, incidences of dyspnea or heart failure, of new $\mathrm{AF}$, and of the composite end point were $57 \% \pm 22 \%, 14 \% \pm 9 \%$, and $75 \% \pm 13 \%$, respectively $(4.3 \%, 2.6 \%$, and $13 \%$ yearly, respectively; Figure 2$)$. Of the posttransplantation patients, one had AF, one underwent tricuspid surgery, and one died because of TR. Follow-up of other posttransplantation patients was uneventful. Excluding them from analysis of outcome yielded similar event rates, with a 10-year incidence of dyspnea or heart failure of $50 \% \pm 25 \%$ and of the composite end point of $77 \% \pm 13 \%$.

Effect on outcome of right-sided chambers. Enlargement was evaluated in 2 subsets of patients, first in patients with initial conservative management $(n=37$; no tricuspid surgery within 3 months of diagnosis) and second in asymp- 


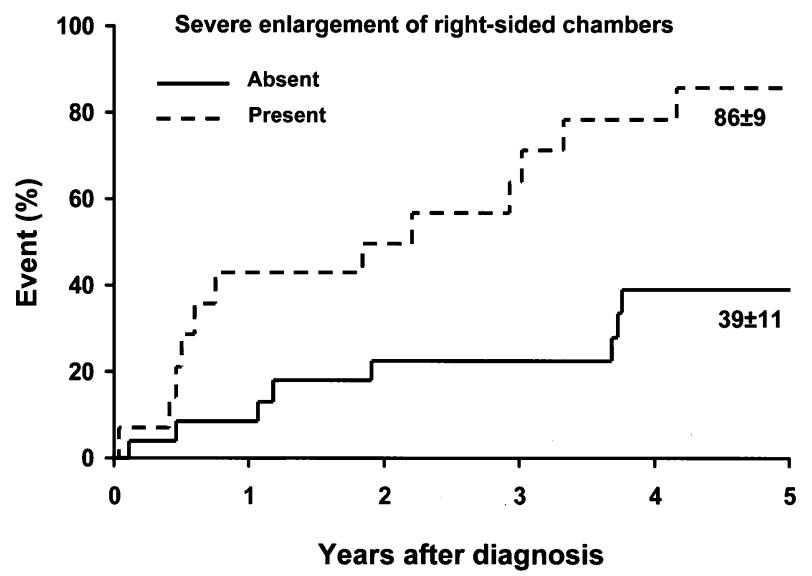

Figure 3. Clinical outcome after diagnosis of TR caused by flail leaflets according to the degree of right-sided chamber enlargement. The Kaplan-Meier curves depict the incidence of the combined end point of symptoms or heart failure, new AF, cardiac surgery, or death in patients initially conservatively managed. Note that patients with severe right-sided chamber enlargement had a higher incidence of events.

tomatic patients (group III). In both subsets the incidence of the composite end point was markedly higher in patients with severe enlargement of right-sided chambers (at 5 years: $86 \% \pm 9 \%$ vs $39 \% \pm 11 \%, P<.01$, Figure 3 ; and $83 \% \pm$ $15 \%$ vs $33 \% \pm 11 \%, P=.08$, respectively). The association of right ventricular fractional area contraction with outcome did not reach statistical significance $(P=.12)$. The higher rates of the composite end point in patients with severe enlargement of right-sided chambers was confirmed in both subsets (hazard ratio and 95\% confidence interval) after adjustment for age, sex, rhythm, and severity of TR (hazard ratio of 1.76 and $95 \%$ confidence interval of 1.12$2.85, P=.02$, in patients with initial conservative management and hazard ratio of 2.39 and $95 \%$ confidence interval of 1.08-6.01, $P=.03$, in asymptomatic patients). Also, the cause (iatrogenic or not) did not influence the outcome in both subsets $(P=.75$ and $P=.51$, respectively).

\section{Natural History}

The natural history of the TR was analyzed in 34 patients in whom the date of occurrence of the tricuspid flail could be specified and excluding group II (associated diseases) patients. The cause was iatrogenic in 14 patients and noniatrogenic in 20 patients. In those 34 patients, the flail leaflet occurred at the age of $38 \pm 24$ years and preceded by $12 \pm$ 16 years the presentation at our institution. Twelve (35\%) patients were seen within 1 year after occurrence of the flail. Twenty-eight (82\%) patients experienced dyspnea, congestive heart failure, or new AF; required tricuspid surgery; or died during the course of the disease. Incidences of symp-

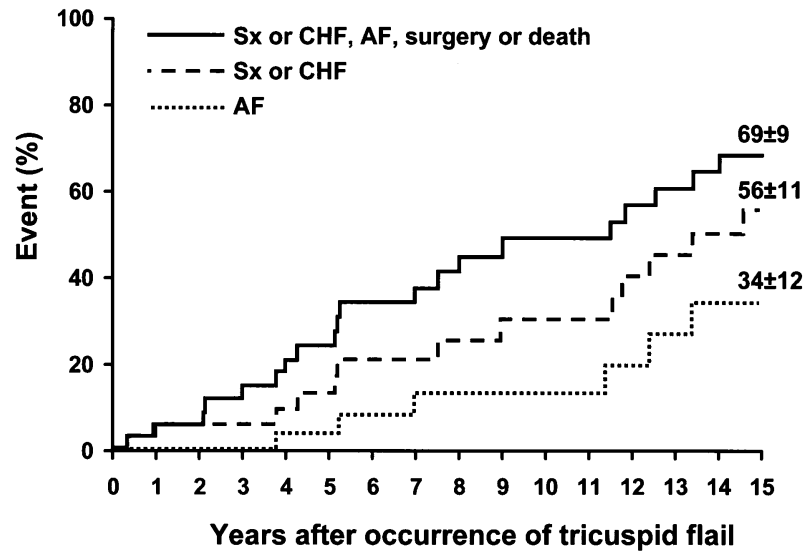

Figure 4. Natural history after occurrence of TR caused by flail leaflets in patients without associated diseases contributing to symptoms. The Kaplan-Meier curves depict the incidence of new AF $(2.8 \%$ yearly), class III or IV symptoms or congestive heart failure (Sx or CHF, $4.4 \%$ yearly), and the composite end point of first occurrence of symptoms or heart failure, new AF, tricuspid surgery, or death $(6.5 \%$ yearly).

toms or heart failure, $\mathrm{AF}$, and the composite end point 15 years after occurrence of the flail tricuspid leaflet were $56 \%$ $\pm 11 \%, 34 \% \pm 12 \%$, and $69 \% \pm 9 \%$, respectively $(4.4 \%$, $2.8 \%$, and $6.5 \%$ yearly, respectively; Figure 4$)$. Even after exclusion of patients undergoing transplantation, the event rate remained high $(69 \% \pm 9 \%)$. The composite end point rate was not different in iatrogenic and noniatrogenic flail leaflets $(79 \% \pm 17 \%$ vs $65 \% \pm 11 \%$ at 15 years, $P=.54)$.

\section{Discussion}

Isolated organic TR is infrequent. We report herein the first sizable series of patients with tricuspid flail leaflets. Our study shows that the cause was mostly traumatic, and the clinical presentation was often severe, despite the usually normal pulmonary pressure. The clinical outcome after diagnosis exhibited excess mortality and high morbidity during follow-up. Even patients asymptomatic at presentation experienced high tricuspid-related event rates, predicted by severe enlargement of right-sided chambers on baseline echocardiography, independent of the cause. This poor outcome was also confirmed by the natural history beginning from the date of occurrence of the flail. Surgical repair of the tricuspid valve is generally feasible, with low operative mortality and marked symptomatic improvement. However, because surgical intervention is mostly performed at an advanced stage, AF is often refractory. Therefore TR caused by flail leaflets is a serious disease characterized over time by a cumulative high rate of events irrespective of its cause, suggesting that an operation should be considered early in its course. 


\section{Clinical Presentation and Outcome}

Isolated organic TR has the reputation of being well tolerated, but there has been no comprehensive study, and case reports have provided contradictory data on outcome. ${ }^{5-11}$ TR caused by flail leaflets has, for outcome analysis, the unique advantages of being easily diagnosed by means of echocardiography and of being frequently associated with large volume overload, despite usually normal right ventricular pressure, ${ }^{13}$ as noted in the present study. Chronic volume overload leads to adverse right atrial, ventricular, and hemodynamic consequences ${ }^{20}$ compounded by occasional right-to-left atrial shunting contributing to hypoxemia. ${ }^{21}$ These adverse consequences lead to functional impairment, ${ }^{22}$ heart failure, and AF. Because TR is often clinically unsuspected, patients are often given a diagnosis at an advanced stage, with frequent symptoms or congestive heart failure, AF, and right-sided chamber dilatation. The serious nature of TR caused by flail leaflets is also demonstrated by the excess mortality observed in comparison with expected mortality. This mediocre outcome is confirmed after exclusion of patients in whom associated diseases could have affected prognosis and in patients asymptomatic at presentation and is independent of the cause of the flail. Asymptomatic patients at presentation exhibited a high incidence of tricuspid-related events after diagnosis, with a 10-year $75 \%$ rate of severe symptoms or heart failure, AF, death, or need for cardiac surgery. We were also able to define the clinical outcome not only from the date of presentation but also from the date of occurrence of the valve lesion. This natural history analysis showed that the 15 -year event rate (symptoms, heart failure, AF, surgical intervention, or death) was high (69\% or $6.5 \%$ yearly), even after exclusion of posttransplantation patients. This rate is particularly important because patients are usually young at occurrence of this mostly posttraumatic lesion, in contrast to the mostly degenerative mitral flail leaflets. ${ }^{23}$ Hence severe complications occur when patients are middle aged and seriously affect their life expectancy. The progression to clinical complications parallels the pattern of volume overloadrelated chamber and annular enlargement, ${ }^{24}$ leading to increasing $\mathrm{TR}^{25}$ and further volume overload. Thus TR caused by flail leaflets is a progressive and serious disease with definite mortality and morbidity risk.

\section{Clinical Implications}

Cause of tricuspid flail leaflets is, contrary to that of mitral flail leaflets, ${ }^{23}$ mainly traumatic. Because clinical diagnosis is difficult, after potential tricuspid trauma, Doppler echocardiography offers the opportunity of early diagnosis ${ }^{14}$ and close follow-up.

The results of our study suggest that early rather than late surgical approach is warranted. Surgical intervention is required in a high proportion of patients, entails low operative mortality and high probability of valve repair, and provides symptomatic improvement. However, because operations are usually performed late in the course of the disease, $\mathrm{AF}$ is often refractory. Therefore in view of the severe consequences of TR caused by flail leaflets, surgical intervention for symptomatic patients should be considered before occurrence of intractable heart failure. For asymptomatic patients, severe enlargement of right-sided chambers predicts poor outcome, and patients with such enlargement should be closely monitored and considered early for surgical intervention. In asymptomatic patients without severe rightsided chamber enlargement, TR and right ventricular dysfunction might be progressive ${ }^{26}$ and close follow-up is warranted. Thus the echocardiographic finding of tricuspid flail leaflets implies a high risk of long-term tricuspidrelated complications and echoes recent data suggesting that severe TR, even of functional cause, might have dire outcome implications. ${ }^{22,27}$ Hence severe TR, particularly that caused by flail leaflets, is a serious and progressive disease, and patients should be warned about the need for close follow-up and be considered early for surgical repair.

\section{Limitations}

The cause of tricuspid flail leaflets is not uniform, but the shared mechanism of TR translates into similar outcomes irrespective of cause. Patients with associated conditions, in whom prognosis might not be totally dependent on the TR, were separately analyzed. Furthermore, complications observed in posttransplantation patients were TR related in the present series. Even when these patients were excluded from analysis, event rates remained high. Therefore cause and associated conditions do not appear to bias the assessment of the TR effect on outcome.

Referral bias cannot be ruled out as affecting the observed outcome. However, a high incidence of complications was also observed in patients asymptomatic at presentation and when the entire natural history was analyzed. Treatment was not randomly assigned, so that the benefit of surgical intervention cannot be definitively proved in asymptomatic patients with severe right-sided chamber enlargement. However, the excellent surgical results with low mortality and high repair rates compare well with the overall excess mortality and the high incidence rates of complications observed in these patients. The frequency of postoperative $\mathrm{AF}$ is, in our opinion, not contradicting the benefit of surgical repair but rather suggesting that patients should be operated on before the occurrence of irreversible cavity enlargement.

Severe right-sided enlargement predictive of outcome was judged from comprehensive multiview echocardiography and confirmed on the basis of monoplane quantitative measurements. The right ventricular function-outcome association, ${ }^{28}$ which was not significant, possibly because of 
volume overload, should be analyzed in future larger series. For that purpose, 3-dimensional quantitative measurement might be useful in patients with TR.

A maze procedure was performed in only 2 patients, and we cannot draw any conclusions. However, such a procedure might usefully complement the tricuspid surgery and needs to be further evaluated.

\section{Conclusion}

TR caused by flail leaflets is most often posttraumatic, and clinical presentation is severe, with outcome characterized by excess mortality and high morbidity. This severe outcome is confirmed by natural history analysis from the time of occurrence of the lesion and suggests that severe TR is a serious disease characterized over time by a cumulative high rate of complications. Tricuspid surgery is often required and can be performed with low operative mortality and high probability of valve repair and symptomatic improvement. Often refractory AF suggests that surgical intervention should be considered earlier before occurrence of intractable heart failure, particularly in patients with dilatation of right-sided chambers.

\section{References}

1. Singh J, Evans J, Levy D, et al. Prevalence and clinical determinants of mitral, tricuspid and aortic regurgitation. Am J Cardiol. 1999;83: 897-902.

2. Tribouilloy CM, Enriquez-Sarano M, Bailey KR, Tajik AJ, Seward JB. Quantification of tricuspid regurgitation by measuring the width of the vena contracta with Doppler color flow imaging: a clinical study. J Am Coll Cardiol. 2000;36:472-8.

3. Koelling TM, Aaronson KD, Cody RJ, Bach DS, Armstrong WF. Prognostic significance of mitral regurgitation and tricuspid regurgitation in patients with left ventricular systolic dysfunction. Am Heart J. 2002;144:524-9.

4. Hung J, Koelling T, Semigran MJ, Dec GW, Levine RA, Di Salvo TG. Usefulness of echocardiographic determined tricuspid regurgitation in predicting event-free survival in severe heart failure secondary to idiopathic-dilated cardiomyopathy or to ischemic cardiomyopathy. Am J Cardiol. 1998;82:1301-3.

5. van Son JA, Danielson GK, Schaff HV, Miller FA Jr. Traumatic tricuspid valve insufficiency. Experience in thirteen patients. $J$ Thorac Cardiovasc Surg. 1994;108:893-8.

6. Almeida M, Canada M, Neves J, et al. Longstanding traumatic tricuspid regurgitation with severe right ventricular failure. J Heart Valve Dis. 1997;6:642-6.

7. Leszek P, Zielinski T, Rozanski J, Klisiewicz A, Korewicki J. Traumatic tricuspid valve insufficiency: case report. J Heart Valve Dis. 2001;10:545-7.

8. Morgan JR, Forker AD. Isolated tricuspid insufficiency. Circulation. 1971;43:559-64.

9. Croxson MS, O'Brien KP, Lowe JB. Traumatic tricuspid regurgitation. Long-term survival. Br Heart J. 1971;33:750-5.

10. Arbulu A, Holmes RJ, Asfaw I. Tricuspid valvulectomy without replacement. Twenty years' experience. J Thorac Cardiovasc Surg. 1991;102:917-22.

11. Arbulu A, Holmes RJ, Asfaw I. Surgical treatment of intractable right-sided infective endocarditis in drug addicts: 25 years experience. J Heart Valve Dis. 1993;2:129-39.

12. Bonow R, Carabello B, DeLeon A, et al. ACC/AHA guidelines for the management of patients with valvular heart disease. Circulation. 1998; 98:1949-84.

13. Tucker PA 2nd, Jin BS, Gaos CM, Radovancevic B, Frazier OH, Wilansky S. Flail tricuspid leaflet after multiple biopsies following orthotopic heart transplantation: echocardiographic and hemodynamic correlation. J Heart Lung Transplant. 1994;13:466-72.

14. Miller FA Jr, Seward JB, Gersh BJ, Tajik AJ, Mucha P Jr. Twodimensional echocardiographic findings in cardiac trauma. Am J Cardiol. 1982;50:1022-7.

15. Hausen B, Albes JM, Rohde R, Demertzis S, Mugge A, Schafers HJ. Tricuspid valve regurgitation attributable to endomyocardial biopsies and rejection in heart transplantation. Ann Thorac Surg. 1995;59:113440.

16. Gayet C, Pierre B, Delahaye JP, Champsaur G, Andre-Fouet X, Rueff $P$. Traumatic tricuspid insufficiency. An underdiagnosed disease. Chest. 1987;92:429-32.

17. McKee P, Castelli W, McNamara P, Kannel W. The natural history of congestive heart failure: the Framingham Study. N Engl J Med. 1971; 285:1441-6.

18. Reddy SC, Rath GA, Ziady GM, Matesic C, Kormos R. Tricuspid flail leaflets after orthotopic heart transplant: a new complication of endomyocardial biopsy. J Am Soc Echocardiogr. 1993;6:223-6.

19. Tunon J, Cordoba M, Rey M, et al. Assessment of chronic tricuspid regurgitation by colour Doppler echocardiography: a comparison with angiography in the catheterization room. Eur Heart J. 1994;15:107484 .

20. Williams MJ, Lee MY, DiSalvo TG, et al. Biopsy-induced flail tricuspid leaflet and tricuspid regurgitation following orthotopic cardiac transplantation. Am J Cardiol. 1996;77:1339-44.

21. Chiu WC, Shindler DM, Scholz PM, Boyarsky AH. Traumatic tricuspid regurgitation with cyanosis: diagnosis by transesophageal echocardiography. Ann Thorac Surg. 1996;61:992-3.

22. Groves PH, Lewis NP, Ikram S, Maire R, Hall RJ. Reduced exercise capacity in patients with tricuspid regurgitation after successful mitral valve replacement for rheumatic mitral valve disease. Br Heart J. 1991;66:295-301.

23. Ling H, Enriquez-Sarano M, Seward J, et al. Clinical outcome of mitral regurgitation due to flail leaflets. N Engl J Med. 1996;335:141723.

24. Iga K, Konishi T, Matsumura T, Miyamoto T, Kijima K, Gen H. Markedly enlarged right atrium associated with physical signs of tricuspid regurgitation - a cause of congestive heart failure in the elderly. Jpn Circ J. 1994;58:683-8.

25. Sagie A, Schwammenthal E, Padial LR, Vazquez de Prada JA, Weyman AE, Levine RA. Determinants of functional tricuspid regurgitation in incomplete tricuspid valve closure: Doppler color flow study of 109 patients. J Am Coll Cardiol. 1994;24:446-53.

26. Mukherjee D, Nader S, Olano A, Garcia MJ, Griffin BP. Improvement in right ventricular systolic function after surgical correction of isolated tricuspid regurgitation. J Am Soc Echocardiogr. 2000;13:650-4.

27. Sagie A, Schwammenthal E, Newell JB, et al. Significant tricuspid regurgitation is a marker for adverse outcome in patients undergoing percutaneous balloon mitral valvuloplasty. J Am Coll Cardiol. 1994; 24:696-702.

28. Zornoff LA, Skali H, Pfeffer MA, et al. Right ventricular dysfunction and risk of heart failure and mortality after myocardial infarction. $J \mathrm{Am}$ Coll Cardiol. 2002;39:1450-5. 